\title{
Electroweak Physics in ATLAS
}

\author{
J. A. Griffiths ${ }^{* \dagger}$ \\ University of Washington, USA \\ E-mail: justin.adam.griffiths@cern.ch
}

\begin{abstract}
Many precision electroweak measurements have been made with the ATLAS detector in protonproton collisions produced by the LHC at $\sqrt{s}=7 \mathrm{TeV}$ in 2010 and 2011. The $W$ and $Z$ boson production cross sections have been measured with electrons, muons, and tau leptons in the final state. Numerous differential cross sections of $W$ and $Z$ bosons with electrons or muons in the final state constrain the uncertainties on the parton distribution functions while also influencing their central values. The $W$ and $Z$ boson cross sections have also been measured in association with a $b$-jet in the final state. The production cross sections for gauge boson pairs: $W W, W Z$, and $Z Z$, have been measured and limits on anomalous triple gauge boson couplings (aTGCs) have been derived.
\end{abstract}

LHC on the March,

November 16-18, 2011

Protvino, Moscow region, Russian Federation

\footnotetext{
* Speaker.

†n behalf of the ATLAS Collaboration
} 


\section{Introduction}

Electroweak measurements provide important tests of the Standard Model predictions at a center of mass energy of $7 \mathrm{TeV}$. In addition, Electroweak and top backgrounds will be the dominant backgrounds for any physics searches beyond the Standard Model. Precision Electroweak measurements give insight into the evolution of the parton distribution functions at high momentum transfer, alignment and calibration of the detector, and an understanding of electron, muon, and tau efficiency and energy scales. All of this information will be crucial for the search of new physics.

Unless stated otherwise, all analyses presented in this proceeding have been performed on data collected in 2010 with a total integrated luminosity of approximately $36 \mathrm{pb}^{-1}$. Analyses summarized in this proceeding include: the $W$ and $Z$ boson production cross sections with electrons, muons, or taus in the final state; $W$ and $Z$ differential cross sections as a function of the gauge boson's $p_{\mathrm{T}}$, the pseudorapidity $(\eta)$ of the lepton from the $W$ boson decay, and the rapidity $(y)$ of the reconstructed $Z$ boson; the cross section of $W$ and $Z$ boson production in association with at least one $b$-jet; and the production cross section for $W W, W Z$, and $Z Z$ gauge boson pairs. More attention will be given to analyses with tau leptons in the final state.

\section{Object Reconstruction}

Many of the analyses discussed in this proceeding share common object selection requirements. Any deviations within individual analyses will be discussed in their respective sections.

Candidate electrons are required to pass either a "medium" or a "tight" [1] cut-based identification and have a $|\eta|<2.47$ excluding the calorimeter barrel-endcap transition region, $1.37<|\eta|<1.52$. "Medium" identification imposes requirements on calorimeter shape variables of the reconstructed cluster, on matching the reconstructed calorimeter cluster to an Inner Detector (ID) track, and imposing quality criteria on the matched track. "Tight" identification, in addition, places more stringent requirements on the matched track, on the energy of the reconstructed cluster vs. the momentum of the matched track, and on hits on the track from the transition radiation tracker. Muons are reconstructed within $|\eta|<2.4$ from a track in the ID matched to a track reconstructed in the Muon Spectrometer using a chi-square matching. The parameters of the combined muon track are derived either from a statistical combination of the parameters of the individual tracks or from a refit of the combined track. The ID track is further required to pass quality criteria including at least one hit in the innermost layer of the pixel detector.

Depending on each analysis, calorimeter and or track isolation will be imposed on electrons or muons. Calorimeter isolation is calculated by summing the transverse energies of all calorimeter deposits within a $\Delta R$ cone about the lepton candidate subtracting any energy associated to the lepton candidate itself. Track isolation is measured by summing the transverse momenta of all charged tracks within a $\Delta R$ cone about the lepton candidate except for the track associated to the lepton candidate itself.

Hadronic tau candidates are required to pass either a "tight" cut-based identification requirement based on the collimation of the tau as measured in the calorimeter and the tracker; or a "tight" boosted decision tree based on the previous variables and on the decay length significance 
of hadronic taus with more than one associated track [2]. Tau candidates are further required to have a $p_{\mathrm{T}}>20 \mathrm{GeV}$ and the same pseudorapidity requirements as the electron candidates.

The transverse missing energy $\left(E_{\mathrm{T}}^{\mathrm{miss}}\right)$ is either reconstructed as the vector sum of all calorimeter energy clusters within $|\eta|<4.5$ corrected for muons; or from all reconstructed and identified physics objects and any additional energy deposits from the remaining calorimeter and tracking information.

\section{3. $W$ and $Z$ Boson Production Cross Sections}

The $W \rightarrow e v$ event selection requires a "medium" identified electron candidate with $p_{\mathrm{T}}>20 \mathrm{GeV}$ and a small value for calorimeter isolation $(\Delta R=0.3)$. Further requirements include: that the $E_{\mathrm{T}}^{\text {miss }}>20 \mathrm{GeV}$ and that the transverse mass $\left(m_{\mathrm{T}}\right)$ of the electron- $E_{\mathrm{T}}^{\text {miss }}$ system is greater than $40 \mathrm{GeV}$. The background from multijet processes is estimated by fitting on the $E_{\mathrm{T}}^{\text {miss }}$ shape where the background template is derived by reversing some of the electron identification variables [3].

The $W \rightarrow \mu v$ event selection requires a muon candidate with $p_{\mathrm{T}}>20 \mathrm{GeV}$ whose isolation, as measured in the tracker within a cone $(\Delta R=0.2)$ is less than $1 \mathrm{GeV}$. Events are further required to have a $E_{\mathrm{T}}^{\text {miss }}>25 \mathrm{GeV}$ and a $m_{\mathrm{T}}>40 \mathrm{GeV}$. The multijet background is estimated in control regions based on the muon's isolation and the $E_{\mathrm{T}}^{\text {miss }}$ [3].

The $W \rightarrow \tau v$ event selection requires a tau with $p_{\mathrm{T}}>20 \mathrm{GeV}$ that passes a "tight" boosted decision tree requirement. Further, the event is required to satisfy $E_{\mathrm{T}}^{\text {miss }}>30 \mathrm{GeV}$ and the missing energy significance is required to satisfy $S_{E_{\mathrm{T}}^{\text {miss }}}=\frac{E_{\mathrm{T}}^{\text {miss }}[\mathrm{GeV}]}{0.5 \sqrt{\mathrm{GeV}} \sqrt{\Sigma E_{\mathrm{T}}[\mathrm{GeV}]}}>6$ where $\Sigma E_{\mathrm{T}}$ is calculated from all calorimeter clusters. The multijet background is estimated from control regions based on whether the hadronic tau candidate passed tight or loose but not tight identification, and on $S_{E_{\mathrm{T}}^{\text {miss }}}[4]$.

Figure 1 shows the transverse mass distributions for all three analyses along with the total measured production cross sections in each of the final states.

The $Z \rightarrow e e$ event selection requires two "medium" identified electrons with $p_{\mathrm{T}}>20 \mathrm{GeV}$, with opposite electric charge, and whose invariant mass, $m_{e e}$, is within $66<m_{e e}<116 \mathrm{GeV}$. The background is estimated by fitting the invariant mass shape, where the background template is derived from data by reversing some of the identification variables of the electrons [3].

The $Z \rightarrow \mu \mu$ event selection requires two muons that pass the same isolation requirement as the $W \rightarrow \mu \nu$ analysis with $p_{\mathrm{T}}>20 \mathrm{GeV}$ and opposite electric charge whose invariant mass, $m_{\mu \mu}$, is required to be within $66<m_{\mu \mu}<116 \mathrm{GeV}$. The multijet background is estimated from control regions based on inverting the isolation requirement on the two muons and on the invariant mass of the two muons [3].

Four final states are considered in the $Z \rightarrow \tau \tau$ analysis depending on how the two tau leptons decay: to hadrons and either a muon $\left(\tau_{h} \tau_{\mu}\right)$ or an electron $\left(\tau_{h} \tau_{e}\right)$, to an electron and a muon $\left(\tau_{e} \tau_{\mu}\right)$, and to two muons $\left(\tau_{\mu} \tau_{\mu}\right)$. Events are triggered by either a single electron or single muon trigger. Electrons are required to have $p_{\mathrm{T}}>16 \mathrm{GeV}$ and to pass either "medium" $\left(\tau_{e} \tau_{\mu}\right)$ or "tight" $\left(\tau_{h} \tau_{e}\right)$ identification. Muons are required to have $p_{\mathrm{T}}>15 \mathrm{GeV}$ if they triggered the event, and $p_{\mathrm{T}}>10 \mathrm{GeV}$ otherwise. Electrons and muons are required to pass tight isolation requirements based both on calorimeter and tracker information. Hadronic tau candidates are required to have $p_{\mathrm{T}}>20 \mathrm{GeV}$ and pass the 

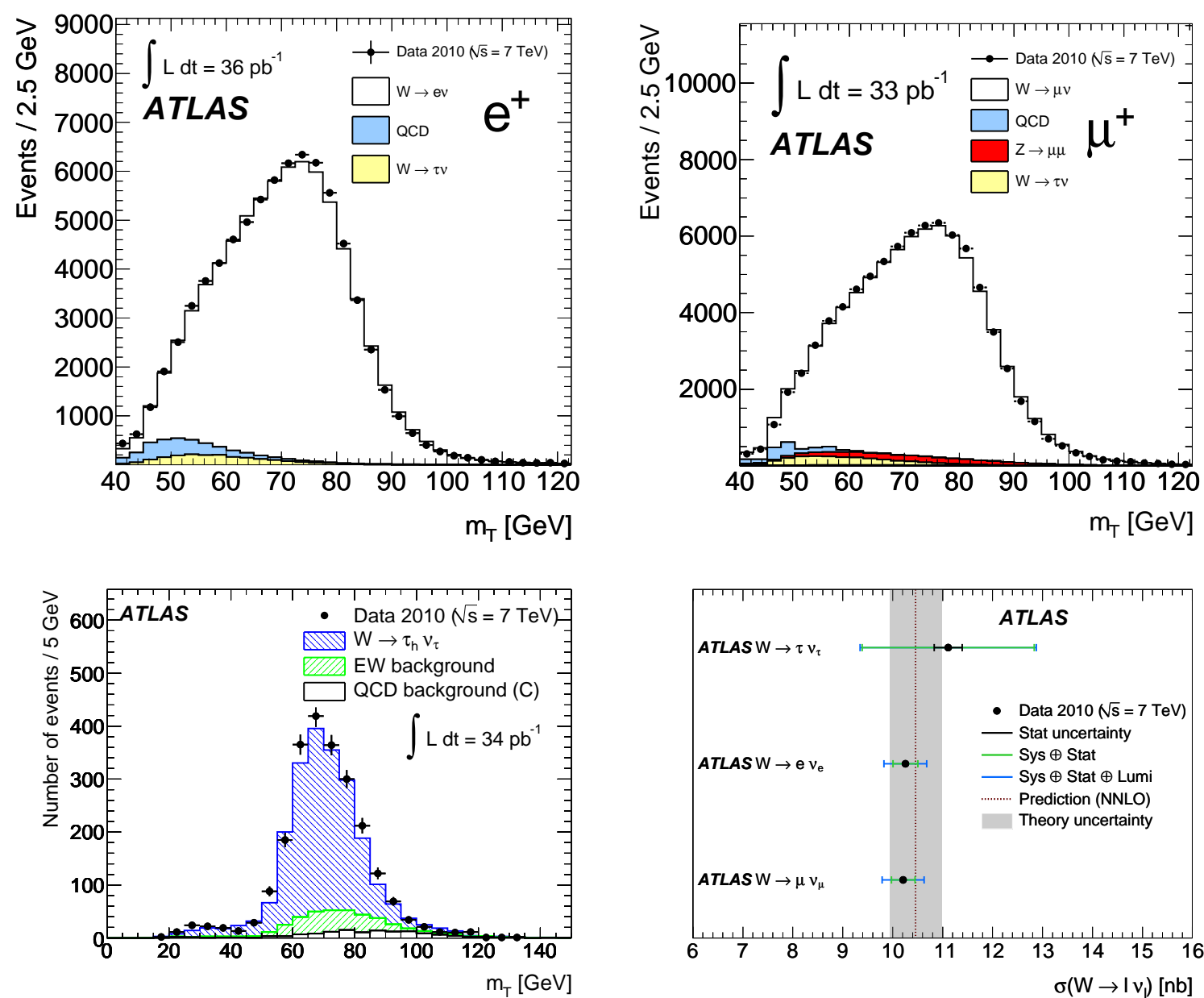

Figure 1: The lepton- $E_{\mathrm{T}}^{\text {miss }}$ transverse masses $\left(m_{\mathrm{T}}\right)$ are shown on top for the $W \rightarrow e^{+} v$ (left) and for the $W \rightarrow \mu^{+} \nu$ (right) analyses. The lower left plot shows the transverse mass for $W \rightarrow \tau \nu$ events where the tau decays hadronically. The lower right plot compares the measured production cross sections for the $W \rightarrow e v$, $W \rightarrow \mu \nu$ and the $W \rightarrow \tau \nu$ channels. The statistical, statistical + systematic, and the total uncertainty are shown for each measurement. The theoretical prediction, along with its uncertainty is depicted with the shaded vertical band.

"tight" cut-based identification requirement. To remove other Electroweak and $t \bar{t}$ processes in the non- $\tau_{\mu} \tau_{\mu}$ final states, only two identified leptons (lepton and identified hadronic tau) are allowed per event, the transverse mass and the total $\Sigma E_{\mathrm{T}}$ is required to be small, and angular correlations between the leptons and the $E_{\mathrm{T}}^{\text {miss }}$ are exploited. The $\tau_{\mu} \tau_{\mu}$ final state uses a boosted decision tree to suppress the overwhelming $\gamma^{*} / Z \rightarrow \mu \mu$ background. All four final states require the two selected leptons to have a reconstructed visible mass $\left(m_{\mathrm{vis}}\right)$ between $35<m_{\mathrm{vis}}<75 \mathrm{GeV}\left(\tau_{h} \tau_{\mu}\right.$ and $\left.\tau_{h} \tau_{e}\right)$, $25<m_{\mathrm{vis}}<80 \mathrm{GeV}\left(\tau_{e} \tau_{\mu}\right)$, and $25<m_{\mathrm{vis}}<65 \mathrm{GeV}\left(\tau_{\mu} \tau_{\mu}\right)$. The multijet background is estimated by splitting events based on whether the two leptons (lepton and hadronic tau) had the same or opposite electric charge and whether the lepton[s] are isolated or anti-isolated. The $\tau_{\mu} \tau_{\mu}$ final state also relies on control regions, but instead bases them on whether the triggered muon is isolated or anti-isolated and whether the second muon is isolated or anti-isolated [5]. 

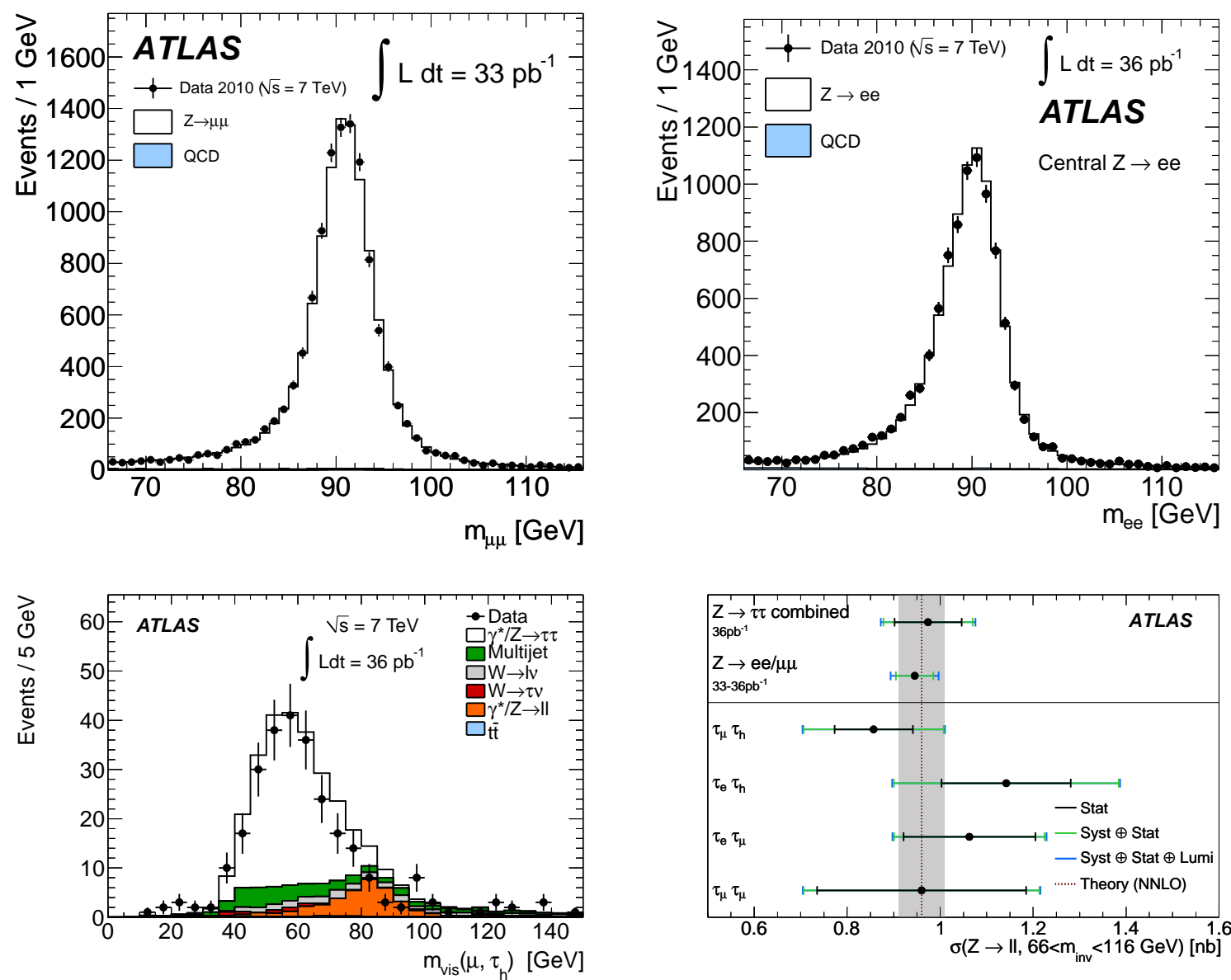

Figure 2: The lepton-lepton invariant masses are shown on top for the $Z \rightarrow e e$ (right) and for the $Z \rightarrow \mu \mu$ (left) analyses. The lower left plot shows the visible mass of the muon and hadronic tau candidate for $Z \rightarrow \tau \tau$ events where one tau decays hadronically while the other tau decays to a muon. The lower right plot compares the measured production cross sections for the combined $Z \rightarrow e e / \mu \mu$ channel and the combined $Z \rightarrow \tau \tau$ channel. In addition, each individual cross section measurement for the four $Z \rightarrow \tau \tau$ final states considered is shown. The statistical, statistical + systematic, and the total uncertainty are shown for each measurement. The theoretical prediction, along with its uncertainty is depicted with the shaded vertical band.

Figure 2 shows the invariant mass of the two selected leptons for the $Z \rightarrow e e, Z \rightarrow \mu \mu$, and $Z \rightarrow \tau \tau$ analyses. In addition, the cross sections are shown for the combined $Z \rightarrow e e / \mu \mu$, the combined $Z \rightarrow \tau \tau$, and the individual $\tau \tau$ final states.

The total experimental uncertainties for the $W / Z$ cross section measurements with electrons or muons are less than $1 \%$, while the experimental uncertainties on the $W \rightarrow \tau v$ and the combined $Z \rightarrow \tau \tau$ cross section measurements are 15 and $6 \%$, respectively. For the non-tau channels, the uncertainty is dominated by the uncertainty on the total integrated luminosity, which is $3.4 \%$. The dominant uncertainty on the $W / Z$ cross sections with taus are due to data statistics, the hadronic tau efficiency, and its energy scale.

$W \rightarrow e / \mu v$ differential cross sections with respect to the reconstructed $W$ boson's $p_{\mathrm{T}}$ and the 

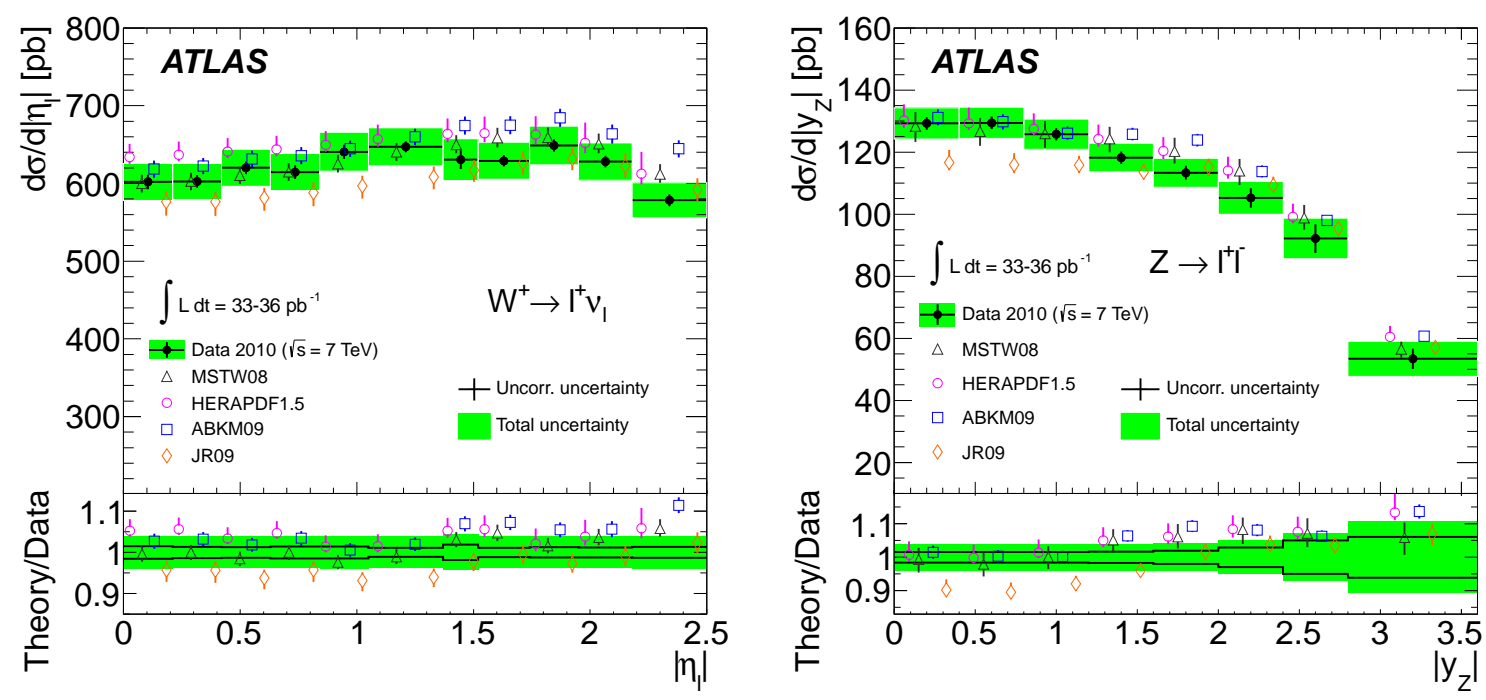

Figure 3: Differential $\mathrm{d} \sigma / \mathrm{d}\left|\eta_{\ell} / y_{Z}\right|$ cross section measurements for the combined $W \rightarrow \ell^{+} v$ (left) $Z \rightarrow \ell \ell$ (right) compared to NNLO theory predictions using various PDF sets [3]. The ratio of theory to data is shown on the bottom of each plot.

$\eta$ of the lepton are measured. In addition, $Z \rightarrow e e / \mu \mu$ differential cross sections with respect to the reconstructed $Z$ boson's $p_{\mathrm{T}}$ and rapidity are measured. The $p_{\mathrm{T}}$ and rapidity of the $Z$ boson are extracted from the sum of the invariant 4-vectors of the two reconstructed leptons [6]. The $p_{\mathrm{T}}$ of the $W$ boson is inferred from the hadronic response of the event after subtracting for the response of the lepton. The measured response of the event is then corrected to the truth level using a response matrix derived from MC [7].

Figure 3 compares the measured differential cross sections of the $W^{+}$, with respect to the pseudorapidity of the lepton, and the $Z$, with respect to its rapidity, to theory using various PDF sets. In general, the experimental accuracy is about $2 \%$ in each bin of $\eta$ and $y$, except in the forward region of the $Z$ analysis where the uncertainties approach 6-10\%. There is broad agreement between theory and experiment, however, there are some discrepancies-especially at low rapidity in the $Z$ analysis. These results should both constrain the theoretical uncertainty on the PDFs and influence their central values.

Figure 4 compares the $Z p_{\mathrm{T}}$ differential cross section to multiple theoretical predictions at NNLO and the data vs. RESBOS prediction for both the $W$ and $Z p_{\mathrm{T}}$ differential cross sections. The latter result is impressive in that it shows that two independent analyses produced similar results with respect to a common theory.

\section{4. $W$ and $Z$ Boson Production in Association with $b$-Jets}

The production cross sections for $W$ and $Z$ bosons in association with at least one $b$-jet have been measured in the electron and muon channels by performing the same event selection as described in section $3[8,9]$. In addition, at least one jet, that is separated from the lepton candidate $(\Delta R($ jet,$\ell)>0.5)$, with $p_{\mathrm{T}}>25$ and $|y|<2.1$ is required. The jets are reconstructed from threedimensional calorimeter clusters that are combined with the anti- $k_{t}$ algorithm using a distance pa- 

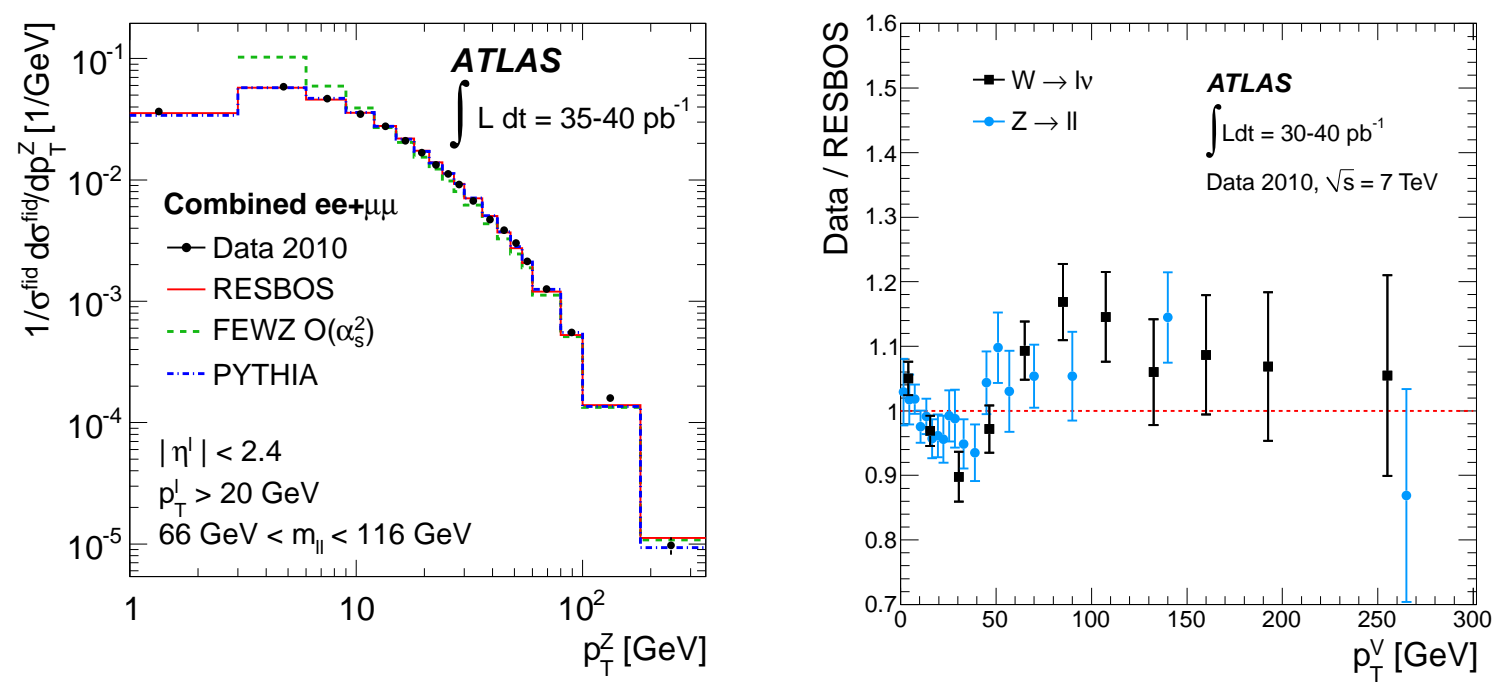

Figure 4: The measured differential cross section $\mathrm{d} \sigma / \mathrm{d} p_{\mathrm{T}}(Z)$ compared with the predictions from RESBOS, PYTHIA, and FEWZ at $\mathscr{O}\left(\alpha^{2}\right)$ (left) and the data/theory (RESBOS) for both the $W$ and $Z$ differential cross sections (right).

rameter $R=0.4$. The jet is then tagged as a $b$-jet if its secondary decay vertex length significance (SV0) is greater than 5.85. After the application of the $b$ tagger, there is still significant background from charm and light flavor quarks. In order to extract the signal, a fit is performed on the mass of the reconstructed secondary vertex where template shapes are derived from MC simulation (fig. 5).

The measured $Z+b$ jet cross section is consistent with the theoretical prediction, but is statistics limited. The measured $W+b$ jet cross section is consistent within $1.5 \sigma$ of the theoretical prediction. Figure 5 shows the measured and predicted $W+b$ jet production cross section in exclusive 1,2 , and $1+2$ jet bins. The measured cross section is systematically higher than the predictions.

\section{Double Gauge Boson Production Cross Sections}

The $W W$ [10], $W Z$ [11], and $Z Z$ [12] dibson production cross sections have been measured in the final states where the bosons decay to electrons, muons, or taus that subsequently decay leptonically. Unlike the other analyses presented in this note, the dataset used in the diboson analyses represents $1.02 \mathrm{fb}^{-1}$. Limits on anomalous (neutral) triple gauge boson couplings are derived in the $W Z(Z Z)$ analysis.

The $W W$ analysis requires two leptons with $p_{\mathrm{T}}>20 \mathrm{GeV}$ that have opposite charges. To remove $Z$ processes when the two leptons are the same flavor, the invariant mass of the lepton pair must not be within $15 \mathrm{GeV}$ of the $Z$ pole. To remove Drell Yan and multijet processes, a modified missing energy requirement is imposed:

$$
E_{\mathrm{T}, \mathrm{Rel}}^{\text {miss }}= \begin{cases}E_{\mathrm{T}}^{\text {miss }} \times \sin \left(\Delta \phi\left(\ell, E_{\mathrm{T}}^{\mathrm{miss}}\right)\right) & \text { if } \Delta \phi<\pi / 2 \\ E_{\mathrm{T}}^{\text {miss }} & \text { if } \Delta \phi \geq \pi / 2\end{cases}
$$



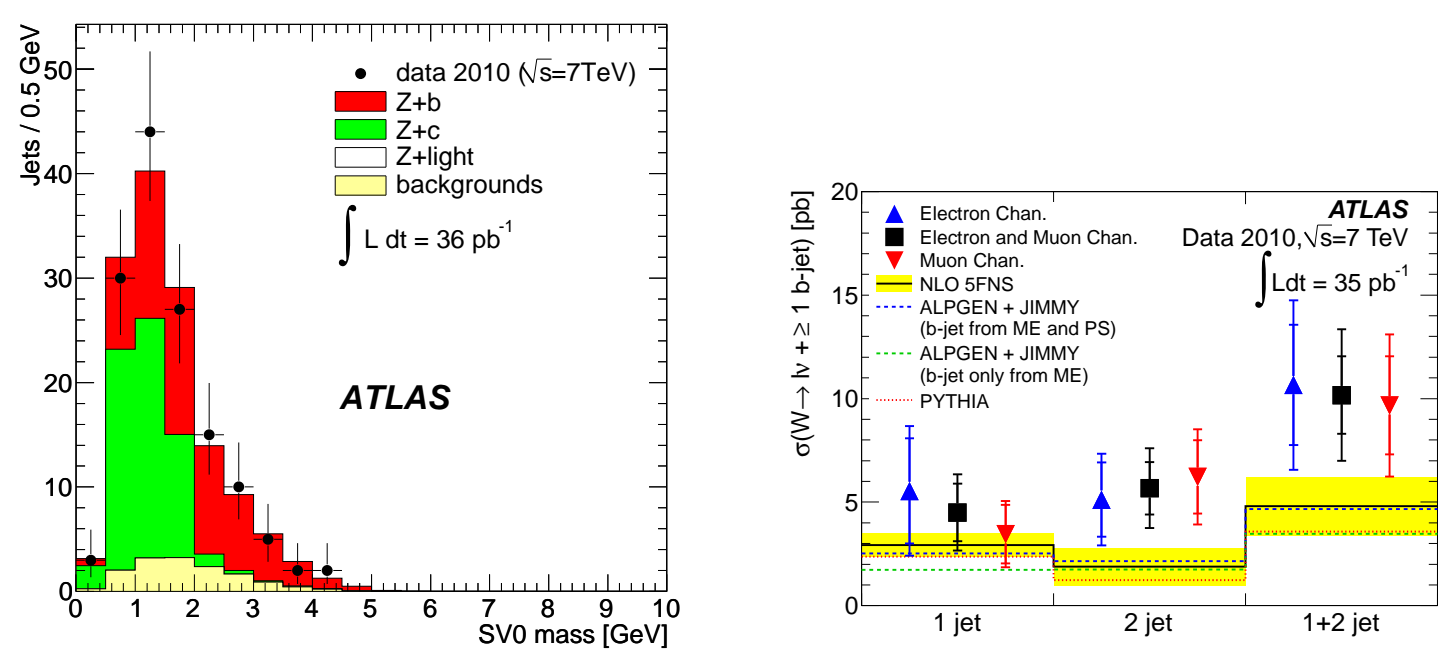

Figure 5: The secondary decay vertex mass is plotted for the candidate $Z \rightarrow e e / \mu \mu+b$ jet events (left). The fitted contributions from light, charm, bottom, and other backgrounds is also shown. The figure on the right shows the measured and NLO theoretical prediction of the $W+b$ jet fiducial cross section in the 1 jet, 2 jet, and $1+2$ jet exclusive bins. The inner uncertainty bar on the data points represent the statistical uncertainty, while the outer band represents the statistical and systematic uncertainty. The shaded band represents the total uncertainty on the theoretical predictions. Theoretical predictions from ALPGEN interfaced with JIMMY and HERWIG are shown with $b$ jet contributions from parton showering and matrix elements (ME and PS) and from matrix elements only (b-jet only from ME). In addition, the prediction from PYTHIA is also shown.

which tends to remove events where the lepton is aligned with the $E_{\mathrm{T}}^{\text {miss }}$. An event with any reconstructed anti- $k_{t}$ jet with $p_{\mathrm{T}}>30 \mathrm{GeV}$ and $|\eta|<4.5$ is removed to suppress $t \bar{t}$ processes. A total of 414 events are observed with an expected background of 170 events. The measured combined cross section is consistent with the NLO theoretical prediction (tab. 1).

\begin{tabular}{|c|c|c|}
\hline Process & Measured cross section & Theoretical NLO cross section \\
\hline $\mathrm{WW}$ & $48.2 \pm 4$ (stat.) \pm 6.4 (sys.) \pm 1.8 (lumi.) $\mathrm{pb}$ & $46 \pm 3 \mathrm{pb}$ \\
$\mathrm{WZ}$ & $20.5_{-2.8}^{+3.1}$ (stat. (1.3. $_{-1.3}^{+1.4}$ (sys. $)_{-0.8}^{+0.9}$ (lumi.) pb & $17.3_{-0.8}^{+1.3} \mathrm{pb}$ \\
$\mathrm{ZZ}$ & $8.5_{-2.3}^{+2.7}$ (stat. $)_{-0.3}^{+0.4}$ (sys.) ${ }_{-0.3}^{+0.3}$ (lumi.) pb & $6.5_{-0.2}^{+0.3} \mathrm{pb}$ \\
\hline
\end{tabular}

Table 1: The measured production cross sections of the double gauge boson processes: WW, WZ, and ZZ, along with the SM expectation calculated at NLO in perturbative QCD.

Four final states are considered in the $W Z$ analysis: eee, $\mu e e, e \mu \mu$, and $\mu \mu \mu$. Two opposite signed same flavor leptons are required to be within $10 \mathrm{GeV}$ of the $Z$ boson mass, while a third lepton, from the $W$, is required to pass more stringent identification and kinematic requirements. In addition, the transverse mass of the $W$ boson, formed from the $E_{\mathrm{T}}^{\text {miss }}$ and the third lepton in the event, is required to satisfy $m_{\mathrm{T}}>20 \mathrm{GeV}$ and the event is required to have $E_{\mathrm{T}}^{\text {miss }}>25 \mathrm{GeV}$. A total of 71 events are observed with an expected background of 12 events. The measured combined cross section is consistent with the standard model prediction (tab. 1). 
Three final states are considered in the $Z Z$ analysis depending on how each $Z$ boson decays: eeee, $е e \mu \mu$, and $\mu \mu \mu \mu$. Two pairs of opposite signed same flavor leptons with $p_{\mathrm{T}}>15 \mathrm{GeV}$ and whose invariant mass satisfies $66<m_{\ell \ell}<116 \mathrm{GeV}$ are required. The lepton which triggered the event is further required to be on the trigger plateau. A total of 12 events are found with an expected background of 0.3 events. The combined measured cross section is consistent with the Standard Model prediction (tab. 1).

\section{References}

[1] ATLAS Collab., Electron performance measurements with the ATLAS detector using the 2010 LHC proton-proton collision data, arXiv:1110.3174; CERN-PH-EP-2011-117, CERN, Geneva, 2011;

[2] ATLAS Collab., Reconstruction, energy calibration, and identification of hadronically decaying tau leptons, ATLAS-CONF-2011-077, CERN, Geneva, 2011.

[3] ATLAS Collab., Measurement of the inclusive $W^{+,-}$and $Z / \gamma$ cross sections in the electron and muon decay channels in pp collisions at $\sqrt{s}=7 \mathrm{TeV}$ with the ATLAS detector, arXiv:1109.5141 [hep-ex]; accepted for publication by Phys. Rev. D.

[4] ATLAS Collab., Measurement of the $W \rightarrow \tau v$ cross section in pp collisions at with the ATLAS experiment, Phys. Lett. B 706, 276 (2012).

[5] ATLAS Collab., Measurement of the $Z \rightarrow \tau \tau$ cross section with the ATLAS detector, Phys. Rev. D 84, 112006 (2011).

[6] ATLAS Collab., Measurement of the transverse momentum distribution of $Z / \gamma^{*}$ bosons in proton-proton collisions at $\sqrt{s}=7 \mathrm{TeV}$ with the ATLAS detector, Phys. Lett. B 705, 415 (2011).

[7] ATLAS Collab., Measurement of the transverse momentum distribution of $W$ bosons in pp collisions at $\sqrt{s}=7 \mathrm{TeV}$ with the ATLAS detector, Phys. Rev. D 85, 012005 (2012).

[8] ATLAS Collab., Measurement of the cross section for the production of a $W$ boson in association with b-jets in pp collisions at $\sqrt{s}=7$ TeV with the ATLAS detector, Phys. Lett. B 707, 418 (2012).

[9] ATLAS Collab., Measurement of the cross-section for b-jets produced in association with a $Z$ boson at $\sqrt{s}=7 \mathrm{TeV}$ with the ATLAS detector, Phys. Lett. B 706, 295 (2012).

[10] ATLAS Collab., Measurement of the $W^{+} W^{-}$production cross section in proton-proton collisions at $\sqrt{s}=7 \mathrm{TeV}$ with the ATLAS detector, ATLAS-CONF-2011-110, CERN, Geneva, 2011.

[11] ATLAS Collab., Measurement of the $W^{ \pm} Z$ production cross section and limits on anomalous triple gauge couplings in proton-proton collisions at $\sqrt{s}=7 \mathrm{TeV}$ with the ATLAS detector, Phys. Lett. B 709, 341 (2012).

[12] ATLAS Collab., Measurement of the ZZ Production Cross Section and Limits on Anomalous Neutral Triple Gauge Couplings in Proton-Proton Collisions at $\sqrt{s}=7 \mathrm{TeV}$ with the ATLAS Detector, Phys. Rev. Lett. 108, 041804 (2012). 